\title{
Chemical Nano-Imaging with Tip-Enhanced Vibrational Spectroscopy
}

\author{
Eric A. Muller ${ }^{1}$ and Markus B. Raschke ${ }^{1}$
}

${ }^{1 .}$ Department of Physics, Department of Chemistry, and JILA, University of Colorado, Boulder, USA

Many organic materials in electronic biological systems gain functionality through molecular structuring, heterogeneous morphologies, chemical interactions and coupling at functional interfaces. A major obstacle to understanding the fundamental nature of intra- inter- molecular interactions, however, is the combination of multi-length scale structural disorder ranging from nanometers to micrometers and multi-timescale dynamical interactions ranging from femtoseconds to minutes.

To gain the desired nanometer spatial resolution with simultaneous spectroscopic specificity we combine scanning probe microscopy with vibrational spectroscopies using both tip-enhanced Raman (TER) and IR scattering scanning near-field optical microscopy (IR s-SNOM). We use TERS and IR $s$ SNOM to probe at the homogeneous sample size limit by virtue of the nanometer spatial near-field localization. Together with the associated enhanced near-field light-matter interaction based on improved mode matching provides domain-level structural information and even single-molecule sensitivity. We present several recent advances in near-field microscopy applied to nanoscale chemical identification, imaging nanoscale defects and the local chemical environment, and observations of dynamical fluctuations at the single molecule limit [1-5].

IR $s$-SNOM has become a powerful tool for nanoscale chemical identification of organic materials. Selected single wavelength IR s-SNOM tuned to the frequency of a characteristic vibrational mode has become a routine method for chemical mapping with nanoscale resolution, while broadband IR s-SNOM can offer greater chemical information through spectroscopic analysis of one or more vibrational modes (Fig. 1A). Using synchrotron infrared nano-spectroscopy (SINS) we extend the spectral range to ultrabroadband nanoscale spectroscopy spanning $300-5000 \mathrm{~cm}^{-1}$ to provide unambiguous nanoscale chemical identification through the vibrational fingerprint [2].

We show an extension of infrared nano-spectroscopy to investigate the structure-function relationship of materials beyond basic chemical identity, using the structural sensitivity of vibrational modes combined with nanoscale spatial resolution. The sensitivity to bond orientation and packing gives access to measures of disorder, structural orientation, and polymorphism. From the symmetry-selective probing of vibrational normal modes we determine phases and orientation in aggregates and thin films of a molecular semiconductor as shown in Fig. 1B. We show nanoscale maps of crystallographic structure in molecular materials through characteristic normal modes using IR $s$-SNOM. We observe nanoscale defects through spatial variation in the molecular orientation of otherwise morphologically well-ordered thin films [3].

Through vibrational solvatochromism, IR $s$-SNOM gives insight into coupled electronic structure, local electric fields, and charge transfer. Vibrational resonances can shift in frequency or change in line width due to modifications in their local chemical environment. Specific marker resonances serve as sensitive probes of highly heterogeneous and coupled molecular systems, within individual nanoscale domains and at interfaces. Using a combination of multispectral s-SNOM imaging with computational image analysis, we map spatial inhomogeneity in long chain block copolymer heterostructures that form disordered quasi-lamellar structures, with significant mixing across the interface. Solvatochromism of the carbonyl mode of PMMA act as a local probe of the chemical environment. Vibrational Stark-shifts 
and correlated broadening of the carbonyl mode within and across individual domains map mixing and heterogeneity on the natural length scales of disorder [4].

Disorder and multi-timescale fluctuations continue to play a role in material properties down to the single molecule limit due to intra-molecular and system-bath coupling. TERS has enabled identification of singe molecules through their vibrational fingerprints, yet a time-averaged or spatially averaged picture of even single molecules blurs any evidence of molecular motions or vibrational dynamics [5]. We measure molecular motions and vibrational dynamics using high spectral resolution TERS at variable and cryogenic temperatures. Suppression of activated dephasing processes lead to long dephasing times and narrow linewidths at low temperatures, and we quantify ultrafast vibrational dephasing through temperature dependent spectroscopy. At cryogenic temperatures, individual hopping events slow to the timescale of seconds, and we observe uncorrelated jumps and spectral diffusion in time series spectra as shown in Fig. 1C. Through statistical correlation analysis of fluctuations of individual modes, can determine instantaneous changes in molecular orientation as well as heterogeneity due to a changing local environment. These results expand TERS from a tool for molecular fingerprinting to a powerful probe of single molecule dynamical processes [5].

Beyond these specific examples of determining composition, structure, and coupling in molecular systems, I will demonstrate the general applicability of vibrational nano-spectroscopy and -imaging for the study of heterogeneity in a wide range of molecular and bio systems as well as the study of solids, organic-inorganic heterostructures, and plasmonic/photonic materials. I will discuss the ongoing extension into the time domain for ultrafast nano-imaging as well as developments for probing under insitu and environmental conditions. I will conclude with a broader perspective of the range of novel nearfield nano-imaging modalities, complementing X-ray and electron microscopy, aiming towards the ultimate goal of obtaining full multi-scale, multi-functional materials characterization.

\section{References:}

[1] Eric A. Muller, Benjamin Pollard, Markus B. Raschke, J. Phys. Chem. Lett., 6 (2015) p. 1275.

[2] Hans A. Bechtel et al, Raschke Proc. Natl. Acad. Sci. USA 111 (2014) p. 7191.

[3] Eric A. Muller et al, Sci. Adv. 2 (2016) e1601006.

[4] Ben Pollard et al,. Commun. 5 (2014) p. 3587.

[5] Kyoung-Duck Park et al, Nano Letters 16 (2016) p. 479.
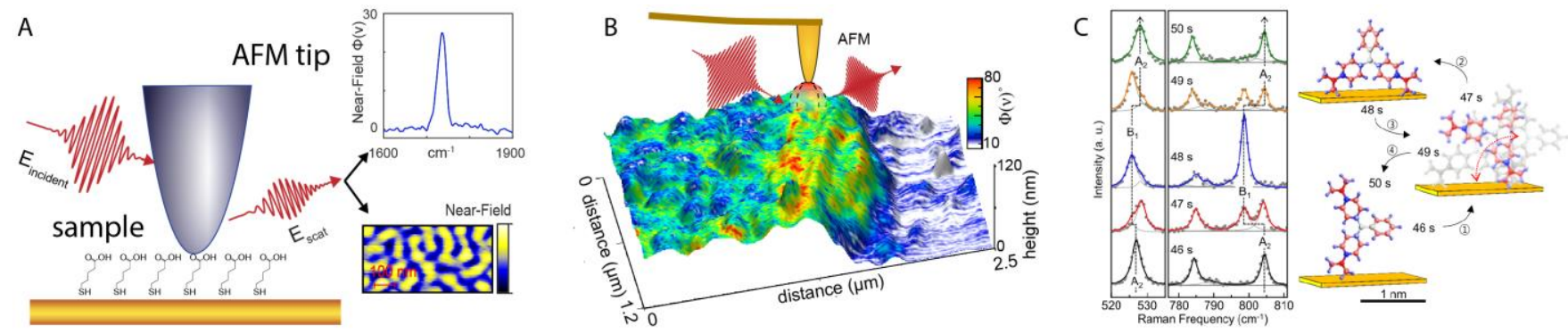

Figure 1. Tip-enhanced/tip-scattering vibrational Raman and IR nano-spectroscopy and -imaging: a) schematic showing tip scattered light with measured vibrational spectrum and nanoscale image [1]. b) SINS and IR s-SNOM measure crystallinity and image orientational defects in a thin film molecular material [2-3]. c) TERS time series spectra measure intramolecular vibrational redistribution and single molecule spectra diffusion [5]. 